\title{
Improved Harmony Search Algorithm with Ant Colony Optimization Algorithm to Increase the Lifetime of Wireless Sensor Networks
}

\author{
Zahra Kamaei \\ Faculty of Computer and \\ Information Technology \\ Engineering, Science and \\ Research Branch, Islamic Azad \\ University, Qazvin, Iran
}

\author{
Hamidreza Bakhshi \\ Associate Professor, Electrical \\ Engineering Department, \\ Shahed University, Tehran, Iran
}

\author{
Behrooz Masoumi \\ Assistant Professor, Faculty of \\ Computer and Information \\ Technology Engineering, \\ Qazvin Branch, Islamic Azad \\ University, Qazvin, Iran
}

\begin{abstract}
The new generations of networks are sensor networks which typically consist of a large number of nodes that are connected wirelessly. The main idea of these types of networks is collecting data around the network's sensors. Since the sensors nodes work with the battery and there is no possibility to change or recharge these batteries, the life time of these networks depends on the sensors energy. The purpose of this study is, improved Harmony Search algorithm with using Ant Colony Optimization Algorithm to determine the basic paths to harmony memory is initialized for increase the life time of the network. For this purpose, an algorithms have been proposed named by ACO-HS also important conditions such as appropriate distribution of energy consumption between sensors nodes leading to increasing life time of the networks, is considered. The simulation results show the capability of the proposed algorithm in finding the Proper path and establishment appropriate balance in the energy consumed by the nodes. Propose algorithm is better than Harmony Search algorithm and Ant Colony Optimization and Genetic Ant Algorithm.
\end{abstract}

\section{Keywords}

Wireless Sensor Network, Routing, Meta heuristic algorithms, Harmony Search Algorithm, Ant Colony Optimization Algorithm.

\section{INTRODUCTION}

A wireless sensor network is a collection of a large number of sensor nodes with small dimensions and by limited capabilities of communications and computing is used to collect and transmit data from the environment to the base station. Despite advances in this type of networks, sensor nodes still to meet their energy requirement depends on the batteries with low power. Therefore, one of the most important issues in wireless sensor networks, is energy efficiency management [1]. Also, since the efficiency of wireless sensor networks is strongly depends on the lifetime of network, in this kind of network protocols should consider the increasing of the lifetime of the network. By definition, the time lapse since the beginning of the network, it takes energy to finish in the first node, called the network lifetime [2]. The purpose of this study, is the combination of Harmony Search Algorithm (HS)[3] and Ant Colony Optimization Algorithm (ACO)[4], as successful meta heuristic algorithms, for routing in wireless sensor networks, in order to increase the lifetime of these networks.

Harmony Search Algorithm is a meta heuristic algorithm that was proposed in 2001. It is inspired based on the form of music orchestra on the methods and operation to achieve coordination between the components. In wireless sensor networks, the harmony search algorithm has been used for problems such as clustering [5], coverage [6], localization [7] and routing [8]. Ant Colony Optimization algorithm was proposed in 1992. It is one of the most apparent examples of collective intelligence that has been inspired by the collective behavior of ants. The Algorithm is used to solve Traveling Salesman problem [9], scheduling [10] and routing [11].

In the proposed algorithm, to reduce energy consumption of the path, it was tried to consider the shortest path between the source node to the destination node with the highest average energy of nodes participating in the route. Thus, if the energy consumed in the route is less and the average residual energy nodes participating in the route is more, the probability of to choose the route increases. It is clear that the routing according to two criteria, is a Multi-objective optimization problem and NP - hard problem that it can be solved by means of an approximation algorithms. The need to establish an appropriate balance between energy consumption and increase the average remaining energy in routing nodes participating is considered effectively in the fitness function of harmony search algorithm. In the next, the introduction of several research similar about energy aware routing at network using wireless Sensor networks by meta heuristic algorithms is considered. At [12], Multi-objective routing at wireless sensor network has done by Genetics Algorithm. This routing, two Criterion of energy and time delay are considered constraints for routing. Method recommended at [12], suggests sudden routing. At [13], One Energy-aware routing at wireless Sensor Networks are offered with the use of Particle Swarm Optimization. At This Research, using one Multi-phase Particle Swarm Optimization Restrictions such as connection and all broadcast constraints are considered.

In [14], other way to increase lifetime with using Nervous networks are presented. This Research with using the clustering sensor nodes, tries to increase lifetime of the network. In $[15,16]$, Multi-objective routing at multimedia wireless sensor networks with the goal of increasing final lifetime of the network using Genetics Algorithm is proposed. In [17], it is tried to present routing method based on 
clustering at wireless sensor networks using combined genetic algorithm and ant community to balance the consumption of energy at the network.

The rest of the paper is organized as follows: Section II describes the perfect harmony search algorithm and Ant Colony Optimization algorithm and introduces the features of this algorithm. The proposed method has been described in detail in Section III and Section IV simulation results are presented. Section V is devoted to summary and conclusion.

\section{INTRODUCTION OF HARMONY SEARCH ALGORITHM AND ANT COLONY OPTIMIZATION ALGORITM}

\subsection{Harmony Search Algorithm (HS)}

The algorithm first proposed by Geem, et al. [3]. Consider a group of musicians in one orchestra. The appropriate combination of composed notes implementation by each musician, at finally converts to beautiful music. Each composition from notes played by musicians, is called harmony. Each harmony after the production, should be checked from the Aesthetics view to be sure that the obtained harmony is the same music or not. Consecutive exercises are conducted until the considered harmony to be created. It is clear that the musicians at any time repeat practice, effort to produce better harmony from view of aesthetics, than previous one. At here it is tried that each of the description listed at about orchestra performance music, fitted with components of Harmony Search Algorithm. Each of the musicians at orchestra music, is one variable in the Harmony Search Algorithm. Each musician in the specific time intervals could perform the notes, each variable is selected from specific intervals. Harmony made at orchestra, is indeed one answer vector. As each harmony at orchestra music after production, should be checked from aesthetics view, each solution at Harmony Search Algorithm also should be evaluated with fitness function. Musicians at each repeat of the exercise, effort to improve the quality of the new harmony, it means that new harmonies, must be better than previous harmonies from the aesthetics views. Every musician in the orchestra music, has three options for playing notes that are:

1) Play the famous notes exactly from his or her memory.

2) Play somewhat similar to the notes (with little change in the notes).

\section{3) Play a random note.}

In the first case, as in a musical orchestra each player can take notes during the past practice has been relatively good aesthetically, plays in the harmony search algorithm, a variable can have a decent amount and is currently selected in memory of the harmony. The position of each variable in all rows of the matrix memory harmony is the same. Therefore, when a variable selects the first case, in fact, one of the variables in the memory column harmony is selected. The probability of selection for variable rate depending on the Harmony Memory Considering Rate (HMCR). In the second case, in a musical orchestra, notes that a player can play in the previous exercises to implement some changes. This is called Pitch Adjustment Rate (PAR). Similarly, the harmony search algorithm, a variable can change slightly the value of the parameter HMCR Selected. About the third case, as a musician in an orchestra music can play random notes, the harmony search algorithm can randomly choose the values of a variable. In the next, for the third case, randomized term is used.

\subsection{Ant Colony Optimization Algorithm (ACO)}

The algorithm was presented by Marco Dorigo [9].The collective Intelligence is rather new approach in the optimization problems that inspired from social behavior of insects and animals, such as ants that were inspired to many methods and techniques.

In the real world, the ants move randomly at first. Ants have different routes to find food in advance and as soon as they find food, they create a trace of pheromone returning to the colony. There pheromone secreted by the ants can increase the probability of selecting a path. After some pheromones on the sun began to evaporate, thus absorbing force fall. Pheromone evaporation is an advantage because of convergence to a local optimum solution is prevented.

Ants move can be considered a fully connected graph. Ants move from the vertices to other vertices to create a solution. Assuming $m$ is the total number of ants and $n$ is the number of vertices $k$ ant move from vertices $i$ to $j$ using a probabilistic approach according to equation (1) is performed [11]:

$P_{i j}^{k}(t)= \begin{cases}\frac{\tau_{i j}^{\alpha}(t) \eta_{i j}^{\beta}(t)}{\sum \tau_{i j}^{\alpha}(t) \eta_{i j}^{\beta}(t)} & j \in \text { allowed }_{k} \\ 0 & \text { otherwise }\end{cases}$

$\mathrm{P}_{\mathrm{ij}}^{\mathrm{k}}(\mathrm{t})$ is probability of Ant $k$ movement from $i$ to $j$ vertices at $t$ moment. Allowed is a collection of vertices that ant $k$ has access to them. $\tau_{i j}$ represents the amount of pheromone stored on edge $(j$ and $i)$ and $\alpha$ and $\beta$ parameters that define the importance of pheromones and heuristic information. $\eta$ is the inverse of the edge length that is calculated by the equation (2) as:

$\eta_{i j=\frac{1}{d_{i j}}}$

Pheromone is sticking on edge that connects the vertices $i$ and $j$ together is for all ants that have created solution, according to equation (3) is updated:

$\tau_{i j} \leftarrow(1-\rho) \cdot \tau_{i j}+\Delta \tau_{i j}$

Where $\rho$ is the rate of evaporation, The $\Delta \tau_{\mathrm{ij}}=\sum_{\mathrm{k}=1}^{\mathrm{m}} \Delta \tau_{\mathrm{ij}}^{\mathrm{k}}$ pheromone cast by ant $k$ when moving from vertices $i$ to $j$ At time $t$ to $t+n$. according to the relationship (4) is calculated as:

$\Delta \tau_{i j}^{k}=\left\{\begin{array}{lr}\frac{Q}{l_{k}} & \text { if ant } k \text { goes thrugh } i, j \\ 0 & \text { otherwise }\end{array}\right.$

Where $Q$ is a constant and $d_{i j}$ is distance between two nodes $i$ and $j$.

\section{THE PROPOSED ALGORITHM}

\section{1. Defaults}

Consider a wireless sensor network with stationary nodes. Each node can have data transfer to nodes in its radio range. The most important challenge is the limited power nodes. 
Each node should transmit its data via an appropriate route (considering the distance, energy consumption, and also the path that will help to increase the lifetime of the network), to the sink. All calculations are done in the sink. Size of the packets is considered identical.

Harmony memory is implemented as a matrix where each row is actually a solution to the problem. Therefore, each row of the matrix as a path between the source (first element) and destination (last element) we consider that the entries in each row, are the numbers of the nodes of the graph. The number of rows in the harmony memory matrix is HMS. Harmony memory matrix has $n+1$ Columns and $n$ is the maximum length of a path. This can be a maximum path length to the number of nodes in the graph. The last column of the matrix is for the storage of the fitness function for each surface. According to the harmony search algorithm, we need all the harmony memory rows are randomly generated. It is important to note that after the production of each rows, the two case of being path of the rows and there is no circle in the path, should be set. For this purpose, the first element is considered the source node. Now randomly select one of the neighboring nodes (nodes which are in radio range), (first basic requirement). By considering the condition, the solution can be solved. Now the selected node is omitted from the set of selected nodes to form the rest of the path, (second essential requirement). The condition prevents building circle around the track. This will continue to be elected to the destination node. If during the course of production, due to both the first and second fundamental requirement, we reached an impasse and the conditions are not applicable, delete the path, and instead the new path is produced. By the strategy outlined above, at the end, we have HMS Number of paths of different lengths without circle. After the production of rows, using the fitness function, the fitness value of each row element specified in the corresponding row, is stored. Fitness function in this algorithm. In a relationship (5) is suggested:

fitness function $=1 /($ Energy consumed along the path) + mean energy of nodes participating in the routing

Considering the fitness function as the above, two criteria will be used to reduce energy consumption and energy distribution to find appropriate solution. The harmony search algorithm has a certain number of repetitions using the randomization of the parameters, HMCR and PAR, a new solution is generated. It is important to note that this new solution is a temporary solution to calculate the fitness function, if harmony is better than the worst in memory, will be replaced that row. Second entries in temporary solution using a three-parameter randomization, HMCR and PAR is generated. Next, a description of each parameter is given.

Parameter HMCR: Assuming that we want to produce the third element of temporary solution using the HMCR parameter. To do so, one of the entries in the third column, we randomly selected memory harmony so that two basic criteria to be met. Note that the element of choice is also an option. In this case, it is possible to reach a state of deadlock, in which case we will act similar to the description given in the random parameter. If the value of this parameter is very small and close to zero is selected, the convergence is slow and if it's too big and close to one, the result is not a good solution, this parameter is usually between $0.5,0.9$ is considered[7,8]. HMCR guarantees the appropriate variables that have been achieved over previous iterations, are always maintained.

Parameter PAR: Before explaining parameter PAR, it is necessary to point out here the way of diagnosis of weights of the edges of a graph. Edge weight between node A and node $B$ in equation (6) is calculated.

Weight between two nodes $\mathrm{A}$ and $\mathrm{B}=$ The remaining energy of B / Distance between two nodes A and B

The method of weighted residual energy of the next node, for a better distribution of energy is ultimately intended to increase the lifetime of the network. Also consider the distance between two nodes, thereby reducing the energy consumption. So in fact the weight is more than one path, the path is more worth it. According to the weighting method and the significance of the residual energy of the next node, it is clear that the graph is a directed graph. The weight of the edge between two nodes in a sweep, it may not be identical. One element of the parameter PAR method is similar HMCR except that the node to replace the node that also satisfy two basic requirements, have a higher weight to the previous element. The PAR parameter to improve the results, will be used. Through parameter PAR, distribution of energy consumption is taken into consideration, which ultimately leads to an increase in the lifetime of the network. Note that if the element produced by the parameter HMCR, there is no choice for PAR, produced from the same node we use the HMCR and go to the next element. The local PAR is used to refine the results. The value of this parameter if it is too small and close to zero is selected, the convergence is slow. They cause a little subspace of the solution space and if the search is too large will cause the algorithm to randomly search function, this parameter is considered between 0.2 and 0.6 [7].

Randomization parameter: This parameter is exactly the same as choosing a random element to generate a path, in order to fill the initial harmony memory that was previously described. This means that when we need to produce a random element, it must comply with the basic requirements, a randomly selected element. Note that the line element of an interim solution, it can not be selected component. The time may come when deadlock is reached, we do not have any choice as to establish two basic conditions, in this case we'll be back step back, to produce the previous element again and continue the rest of the production of the path. Randomization is used to increase the diversity of solutions. Although PAR algorithms to local search, and entrapment in local optimum is guided, the use of randomized algorithms into a nationwide search to find the global optimal solution is used.

\subsection{The proposed algorithm (ACO-HS)}

The main idea is improved Harmony Search algorithm with using Ant Colony Optimization Algorithm to determine the basic paths to harmony memory is initialized for increase the life time of the network. How to implement this algorithm is shown in Figure 1. 


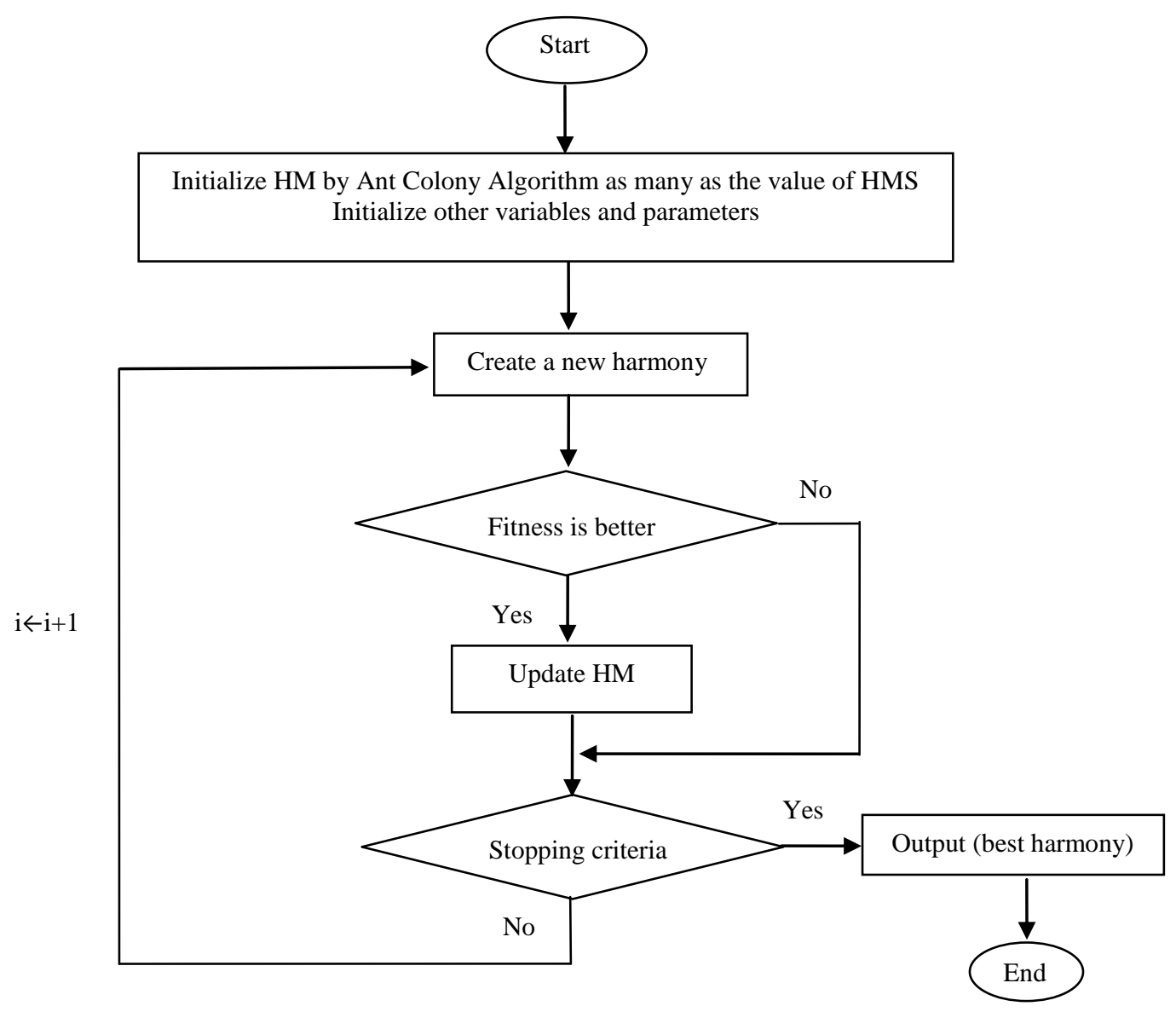

Fig 1: Algorithm performance graph ACO-HS

First parameters and variables are initialized. HM contains the matrix of HMS path that created by Ant Colony Optimization Algorithm. Then the fitness function according to equation (5) is calculated for each path. By using memory consideration, pitch adjustment, and randomization, create a new harmony. If a new harmony's fitness is better than the worst harmony's fitness in the HM, update the HM. These procedures are repeated until the stopping criteria are satisfied.

\section{SIMULATION AND ANALYSIS OUTPUTS}

We consider Wireless sensor network as a graph. The uniform distribution of nodes, the space is divided into several segments and nodes are distributed randomly in the area. Distance information between nodes is stored in a matrix. This matrix can be used to awareness of the connection matrix used to establish a connection between any two nodes. This is necessary for the element that is greater than the maximum radio range of sensors, such as number (-1) can be placed. So if there is the number $(-1)$ between two nodes, the two nodes are not connected and cannot directly submit the data. As mentioned above, the maximum radio range of sensors is considered the same as a matrix to store Energy levels of the nodes is considered that the routing information is updated in the matrix. Energy consumption in this study according to [5] it is intended that the relationship (7) is:

$$
\begin{aligned}
& E_{T}=E_{\text {elec }} * l * E_{f s} * l * d^{2}, \quad d<d_{0} \\
& E_{T}=E_{\text {elec }} * l * E_{f s} * l * d^{4}, \quad d>d_{0} \\
& E_{R}=E_{\text {elec }} * l \\
& d_{0}=\sqrt{E_{f s} / E_{m p}}
\end{aligned}
$$

$E_{T}$ Energy consumption for nodes sender information. $E_{\text {elec }}$ is the energy required to transmit one bit of information that does not depend on distance. $E_{f s}$ and $E_{m p}$ is energy required to transmit a bit of free space model and multi-path fading model. $l$ is the length of the message. $d$ sender node to destination node distance recipient information. $E_{R}$ Energy consumption to the node is receiving data. Each sensor node has both the sending and receiving information. The recipient information to receive a message bits by $E_{\text {elec. }}$ Energy consumption. The transmission has two parts. The first part is to send each bit of information, the amount of energy $E_{\text {elec }}$. Second, the signal amplifier to amplify the desired information over distance. In all simulations the values in Table 1 were used: 
Table 1. The initial values of the parameters

\begin{tabular}{|c|c|}
\hline Parameter & Amount \\
\hline Count Node & 70 Node \\
\hline Perimeter Area & 200 Meter At 200 meters \\
\hline Coordinates well & $190 \mathrm{~m}$ to $190 \mathrm{~m}$ \\
\hline Primary energy nodes & Random in the interval [ $0.4-0.5]$ \\
\hline HMCR & Random in the interval [0.5- 0.9$]$ \\
\hline PAR & Random in the interval $[0.2-0.6]$ \\
\hline $\begin{array}{c}\text { The number of ACO } \\
\text { repetitions }\end{array}$ & 100 \\
\hline $\begin{array}{c}\text { The number of ACO-HS } \\
\text { repetitions for each } \\
\text { routing }\end{array}$ & 300 \\
\hline The number of routing & 1762 Away \\
\hline HMS & 100 \\
\hline$\alpha$ & 0.8 \\
\hline$\beta$ & 1 \\
\hline$\rho$ & 0.8 \\
\hline$E_{f s}$ & $10 \mathrm{pJ} / \mathrm{bit} / \mathrm{m}^{2}$ \\
\hline 1 & $4000 \mathrm{bit}$ \\
\hline$E_{\text {elec }}$ & $50 \mathrm{~nJ} / \mathrm{bit}$ \\
\hline$E_{m p}$ & $0.0013 \mathrm{pJ} / \mathrm{bit} / \mathrm{m}^{4}$ \\
\hline
\end{tabular}

If the fitness function for harmony search algorithm, in conjunction (8), regardless of the average residual energy of the nodes of the sensor and only consider the energy consumption along the path, consider the harmony search algorithm similar to a shortest path algorithm operates as Dijestra $^{1}$ and shortest path between source and destination nodes can be.

fitness function $=1 /$ Energy consumed along the path

With regard to the fitness function according to Equation (8), only the consumption of energy and other parameters considered appropriate distribution of energy consumption in the lifetime of the network is very influential is ignored. To consider the distribution of energy consumption, with the average residual energy of nodes in sensor fitness function using equation (5), then the simulation should be done. By comparing the result of the above-mentioned case the graph example, the standard distribution of energy consumption among sensor nodes, in addition to taking the sample, the energy consumption, it becomes apparent. First, harmony search algorithm on the graph model, only with regard to energy consumption and the latter, try to create a balance between energy consumption and distribution of energy consumption between nodes participating in the routing. The result of these comparisons are shown in Table 2.

\footnotetext{
${ }^{1}$ Dijkstra's algorithm is an algorithm for finding the shortest paths between nodes in a graph.
}

Table 2. Comparison of Harmony search algorithm proposed relationship between the fitness function (5) and Eq. (8)

\begin{tabular}{|c|c|c|c|c|c|}
\hline \multicolumn{2}{|c|}{ Number of nodes } & 40 & 50 & 70 & 100 \\
\hline \multirow{2}{*}{$\begin{array}{l}\text { Lifetime } \\
\text { Network }\end{array}$} & $\begin{array}{c}\text { Relation } \\
(8)\end{array}$ & 1078 & 1138 & 1229 & 1469 \\
\hline & $\begin{array}{c}\text { Relation } \\
(5)\end{array}$ & 1584 & 1612 & 1894 & 1814 \\
\hline \multirow{2}{*}{$\begin{array}{c}\text { After the } \\
\text { death of the } \\
\text { first node } \\
\text { average } \\
\text { energy(Joules) }\end{array}$} & $\begin{array}{c}\text { Relation } \\
(8)\end{array}$ & 0.1074 & 0.1023 & 0.0941 & 0.0982 \\
\hline & $\begin{array}{c}\text { Relation } \\
\text { (5) }\end{array}$ & 0.1202 & 0.1145 & 0.1055 & 0.1138 \\
\hline \multirow{2}{*}{$\begin{array}{c}\text { Standard } \\
\text { deviation of } \\
\text { the residual } \\
\text { energy of the } \\
\text { network } \\
\text { The death of } \\
\text { the first node }\end{array}$} & $\begin{array}{c}\text { Relation } \\
(8)\end{array}$ & 0.098 & 0.101 & 0.092 & 0.094 \\
\hline & $\begin{array}{l}\text { Relation } \\
\text { (5) }\end{array}$ & 0.086 & 0.089 & 0.083 & 0.093 \\
\hline
\end{tabular}

To compare the two cases mentioned above, the three categories of network lifetime, energy consumption and the average standard deviation of residual energy of sensor nodes, after the death of the first node in each case is considered. Whatever the distribution of energy consumption among sensor nodes should be performed, the standard deviation of the residual energy of the nodes of the sensor is less. As can be seen in the latter case, the average increase in energy consumption, the standard deviation is reduced, so in this case, a proper balance between the two measures of energy consumption and distribution of energy, lifetime of the network has been increased. In the second case, the average power consumption is more, but considering the power of participating nodes in routing, the fact of such nodes for routing need more energy. This would increase the network lifetime as well.

To assess the ability of the proposed algorithm, a simulation using harmony search algorithm, Ant Colony Optimization algorithm and Genetic Ant algorithm [17] is performed and the results were compared with each other. Four algorithms are implemented in the same circumstances. Table 3, results and Figure 2 shows the distribution of performance and energy consumption of the routing for four algorithm at 70 nodes.

Table 3. Comparison of algorithm

\begin{tabular}{|c|c|c|c|}
\hline Algorithm & $\begin{array}{c}\text { Lifetime } \\
\text { Network }\end{array}$ & $\begin{array}{c}\text { Standard } \\
\text { After 1762 } \\
\text { the average } \\
\text { energy } \\
\text { consumption } \\
\text { routing (J) }\end{array}$ & $\begin{array}{c}\text { residual energy of } \\
\text { deviation of the } \\
\text { sensor nodes } \\
\text { around 1762 after } \\
\text { routing }\end{array}$ \\
\hline ACO-HS & 1902 & 0.124 & 0.080 \\
\hline ACO [11] & 1762 & 0.98 & 0.099 \\
\hline HS [8] & 1894 & 0.105 & 0.083 \\
\hline $\begin{array}{c}\text { Genetic Ant } \\
\text { [17] }\end{array}$ & 1886 & 0.110 & 0.087 \\
\hline
\end{tabular}

Figure 2 illustrates the fact that the distribution of energy consumption algorithm ACO-HS, relative to harmony search algorithms, ACO algorithm and Genetic Ant algorithm is better than them. ACO-HS algorithm with spend more energy, effort energy distribution, which ultimately leads to increased 
lifetime. However, the ACO-HS algorithm to devote more energy, trying to delay the death of the first node Life expectancy is increasing, but it makes life a network, assuming replacement of dead nodes, the death of the other nodes occur more rapidly.

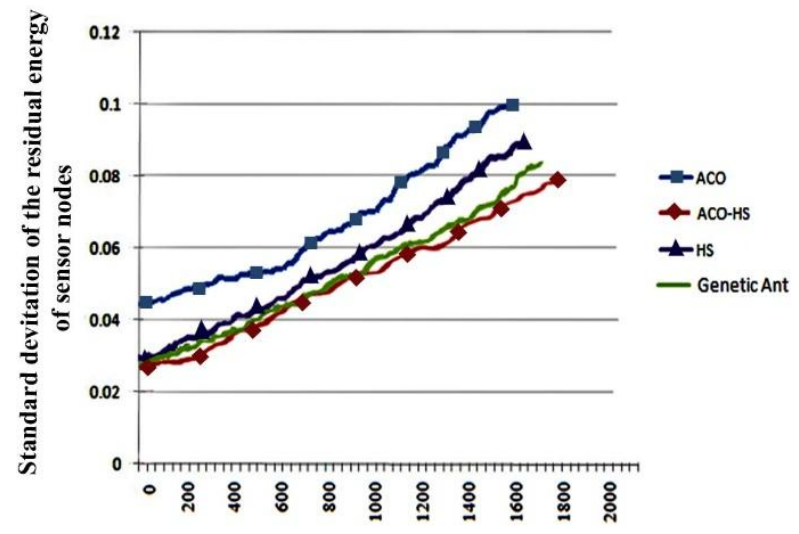

Number of routing

\section{Fig 2. How Energy distribution after the routing}

Figure 3 shows life trend of the network. In the ACO-HS algorithm, due to more energy consumption to increase the lifetime of the network, the lifetime of the whole network has reduced and the numbers of the live nodes has reduced in each moment. Therefore, in applications such as monitoring of the weather that delayed the death of the first sensor node is critical, ACO algorithm and Genetic Ant algorithm works better. Instead of monitoring or tracking applications that postponing the first death of the last death, ACO-HS algorithm, is the right choice and work better. Ant Colony Optimization algorithm, harmony search and genetic ant colony to find the right answer, Need more generation. So in the same environmental conditions and taking into account the number of times of the generation, installation of the Harmony search algorithms and algorithms ACO-HS, fewer quality solutions (the lower the fitness function) is produced. The solution produced by the ACO-HS algorithm with the same generation, in terms of energy consumption and energy distribution is preferred that led to this result.

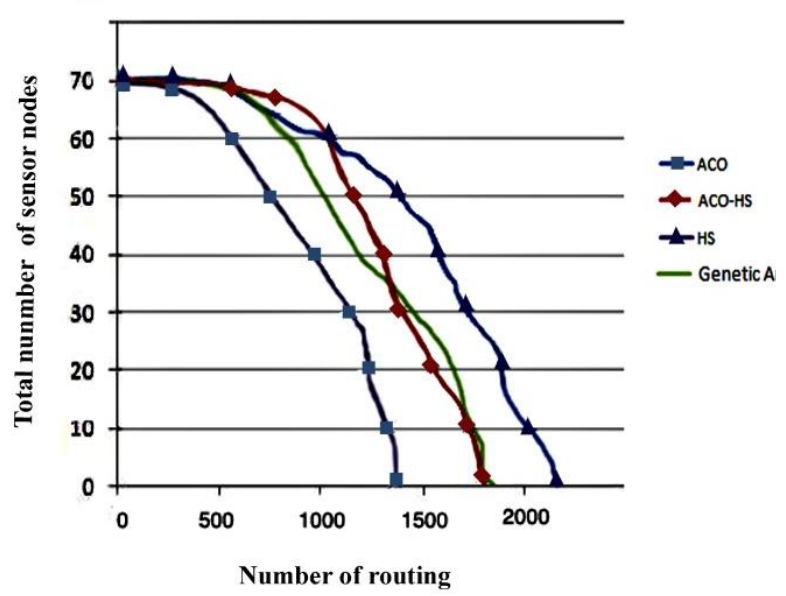

Fig 3. The life trend of the entire network

\section{CONCLUSION}

Result Simulations show that the ACO-HS algorithm is able to create a good balance between energy consumption and increase energy intermediate nodes participating in the routing find better path between the source node and the destination node. The result Simulations show that the ACO-HS algorithm with respect to the HS algorithm, ACO algorithm and Genetic Ant algorithm on equal terms, increase the network lifetime. ACO-HS algorithm uses more energy sensor nodes to routing and creates appropriate distribution of energy consumption by the sensors that increases the lifetime of the network. To increase the average residual energy of sensor nodes participating in the routing which leads to a better distribution of the energy consumption and increase its lifetime and consume more energy. This makes the overall network energy is reduced and the life after death, the first network node, the death rate of the sensor nodes become faster. The number of nodes in each moment of life is reduced and the lifetime of the network decreases. This feature makes the ACO-HS algorithm unlike the ACO algorithm, are important for applications in which the death of the last node is not a good performance.

\section{REFERENCES}

[1] Akyildiz, IF; Su, W.; Sankarasubramaniam, Y.; Cayirci, E.; "A Survey on Sensor Networks"; IEEE Commun. Mag; 40 (8), 2002, 102-114.

[2] Anisi, M .; Bdullah, A .; Azak, R .; Asri Ngadi, M .; " An Overview of Data Routing Approaches for Wireless Sensor Networks "; Sensors; 12, 2012, 3965- 3403.

[3] Geem, ZW; Kim, JH; Loganathan, GV.; "A New Heuristic Optimization Algorithm: Harmony Search"; SIMULATION: Transactions of the Society for Modeling and Simulation International; 76 (2), 2001, 6068.

[4] Dorigo, M.; "Learning and Natural Algorithms"; PHD Thesis; Department of Electronics; Politecnico di Milano; Italy, 1992.

[5] Hoang, DC; Yadav, P.; Kumar, R.; Panda, SK.; " A Robust Harmony Search Algorithm Based Clustering Protocol for Wireless Sensor Networks "; IEEE International Conference on Communications Workshops (ICC); 2010, 1-5.

[6] Ebrahim Nezhad, S .; Kamali, HJ; Moghaddam, ME.; "Solving K-Coverage Problem in Wireless Sensor Networks Using Improved Harmony Search"; International Conference on Broadband; Wireless Computing, Communication and Applications (BWCCA); 2010, 49-55.

[7] Manjarres, D .; Ser, JD; Lopez, SG; Vecchio, M ; Torres, IL; Valcarce, RL; "On the Application of a Hybrid Harmony Search Algorithm to Node Localization in Anchor-based Wireless Sensor Networks"; International Conference on Intelligent System Design and Application (ISDA); 2011, 1014-1019.

[8] RezaeiNejad, M.; Mousavi, SA.; RahimiNasab, M.; "Energy-Aware Routing Using Harmony Search Algorithm"; Journal of Soft Computing; 1, 2012, 2-15.

[9] Dorigo, M.; "Ant Colony System: A Cooperative Learning Approach to the Traveling Salesman Problem"; IEEE Transactions on Evolutionary Computation; 1, 1997, 53-66, 
[10] Zhang, J.; Hu, X.; Tan, X.; Zhong, JH; Huang, Q.; "Implementation of an Ant Colony Optimization Technique for Job ShopScheduling Problem "; Transactions of the Institute of Measurement and Control; 28, 2006, 93-108.

[11] Guo, h.; "Investigation on Ant-Colony Based Routing Algorithm for Wireless Sensor Networks"; Conference on Dependable Computing; 2010, 227-229.

[12] Pourkabirian, A.; Haghighat, AT.; "Energy-aware, Delay-Constrained Routing in Wireless Sensor Networks through Genetic Algorithm"; 15th International Conference on Software; Telecommunications and Computer Networks; 2007, 1-5.

[13] Yuan, P .; Ji, C .; Zhang, Y .; Wang, Y .; "Optimal Multicast Routing in Wireless Ad Hoc Sensor Networks"; International Conference on Networking; Sensing \& Control; 1, 2004, 367-371.

[14] Nehra, NK; Kumar, M; Patel, RB.; "Neural Network Based Energy Efficient Clustering and Routing in Wireless Sensor Networks"; First International
Conference on Networks and Communications; 2009, 34-39.

[15] Niansheng, C.; Zhi, L.; Zongwu, K.; Xiaoshan, G.; "A QoS Multicast Routing Algorithm Based on Genetic Algorithm of Game Selection"; Ninth International Symposium on Distributed Computing and Applications to Business, Engineering and Science; 2010, 308-311.

[16] Dong, W; Ke, Z; Chen, N; Sun, Q.; "QoS Routing Algorithm for Wireless Multimedia Sensor Networks"; 4th International Symposium on Advances in Computation and Intelligence; 2009, 512-524.

[17] Xirong, B; Shi, Z; Dingyu, X.; "Research and Simulation on Genetic Ant Colony Routing in Wireless Sensor Network";4th International Conference on Wireless Communications; Networking and Mobile Computing; 2008, 1-5

[18] Heinzelman, WR; Chandrakasan, AP; Balakrishnan, H.; "An Application- Specific Protocol Architecture for Wireless Micro Sensor Networks"; IEEE Transactions on Wireless Communications; 4 (1), 2002, 660-670. 